\title{
Properties of horizontal flows inside and outside a solar pore
}

\author{
Th. Roudier ${ }^{1}$, J. A. Bonet ${ }^{2}$, and M. Sobotka ${ }^{3}$ \\ ${ }^{1}$ Institute Laboratoire d'Astrophysique de Toulouse, Observatoire Midi-Pyrénées, 14 avenue E. Belin, 31400 Toulouse, France \\ 2 Instituto de Astrofísica de Canarias, 38205 La Laguna, Tenerife, Spain \\ 3 Astronomical Institute, Academy of Sciences of the Czech Republic, 25165 Ondřejov, The Czech Republic
}

Received 17 April 2002 / Accepted 16 August 2002

\begin{abstract}
Horizontal velocities and their temporal variations inside a large pore and in the surrounding granulation are studied from a 73 min sequence of white light frames, acquired at the Swedish Vacuum Solar Telescope (La Palma). The local correlation tracking technique with high spatial $(0.31)$ and temporal $(5 \mathrm{~min})$ resolution was applied to binarized images, yielding 14 independent velocity maps. A ring of divergence centres around the pore was observed in all the maps. Motions directed into the pore, deposited by the divergence centres, continue also within the pore but with magnitudes smaller by factor of 2-3. A link between the variations of large velocity amplitudes around the pore and the brightness fluctuations of umbral dots is suggested. A phase delay between velocity and intensity changes at the periphery of the pore, probably related to the penetration of bright features inwards across the pore's border, was observed.
\end{abstract}

Key words. Sun: granulation - Sun: photosphere - Sun: sunspots

\section{Introduction}

The interaction between plasma and the magnetic field on the solar surface is an important topic in solar physics. This kind of interaction can be studied on different scales ranging from small magnetic flux tubes $(150 \mathrm{~km})$ up to large complex sunspots (80 $000 \mathrm{~km}$ or more). The aim of this study is to learn more about solar pores, structures corresponding to the first stage in the development of sunspots. As solar pores do not show noticeable penumbrae (see Keil et al. 1999 for a recent review on pores), the direct interaction, without intermediate structures, between the vertical magnetic field inside the umbra and the surrounding solar turbulent convection can be studied. The observation of bright granules moving inside a pore by Sobotka et al. (1999) shows the complex exchange between the pore and the surrounding photosphere.

In this paper, we use local correlation tracking techniques (hereafter LCT) applied to segmented binarized images, as described in Roudier et al. (1999), to study the behaviour of the flows inside and outside a solar pore with high spatial and temporal resolution and to extend the previous analyses published by Sobotka et al. (1999) and Hirzberger et al. (2002). The unusually large size of this pore and the good quality and duration of the time sequence of images $(73 \mathrm{~min}$ ) make it appropriate for our purpose.

From our analysis, we are able to describe the continuity of the motion on the solar surface through the boundaries of the pore and also the exploding granule activity in the granular

Send offprint requests to: Th. Roudier,

e-mail: roudier@obs-mip.fr light bridge present in the pore. Despite the limitations of the LCT method (see Simon et al. 1995), we show the influence of the surrounding plasma motions on the evolution of small-scale structures inside the pore.

\section{Observations and data reduction}

Our data consist of a time series of white-light images ( $\lambda 5425 \pm$ $50 \AA$ ) of the active region NOAA 7886 with a group of solar pores located at $\mu=0.92$. This active region appeared on 1995 June 29 and faded on July 3, reaching its maximum development on June 30. The series was recorded at the Swedish Vacuum Solar Telescope on La Palma (see Scharmer et al. 1985) on June 30. The best image within an interval of $15 \mathrm{~s}$ was automatically selected in real time by means of a frame selection algorithm based on the granulation contrast. Considering the time needed to store the selected image on a hard disk, the mean time elapsed between consecutive images was about $20 \mathrm{~s}$. The resulting series contains 220 frames, taken with a typical exposure time of $11 \mathrm{~ms}$, and an image scale of $0{ }^{\prime} 062 \mathrm{pix}^{-1}$.

After the pre-treatment (dark current subtraction and flatfielding), the images were aligned and derotated to compensate for defective tracking and the rotation due to altazimuthal tracking of the Sun.

Before applying any correction, the granulation contrast, as computed in a subfield of undisturbed photosphere, typically amounts to $7.6 \%$ and many images show features with sizes close to the resolution limit of the telescope $(0,24)$ at the working wavelength. The deconvolution of the instrumental profile (assuming a diffraction limited telescope) and noise filtering 
was performed by applying a Wiener filter. The stray light in our measurements can be considered as a minor effect $(\leq 1 \%$ of the mean photospheric intensity, as derived from previous observations obtained with the same instrument and under similar conditions of transparency, wavelength, and seeing).

At this step of the process, a subfield of $18^{\prime \prime} \times 18^{\prime \prime}$ $(288 \times 288$ pixels $)$ including the largest pore of the group was extracted throughout the time series for further processing. To correct for image distorsion, the images of the resulting datacube were destretched using the local correlation routines written by Molowny-Horas \& Yi (1994), based on the paper by November \& Simon (1988).

Finally, we applied a subsonic filter with a cut-off phase velocity of $4 \mathrm{~km} \mathrm{~s}^{-1}$ to the datacube to remove the acoustic modes and the residual jitter in the movie that remained after the destretching process. Figure 1 shows one of the best resulting images of the pore after applying all the corrections. A more detailed description of the data reduction process can be found in Sobotka et al. (1999).

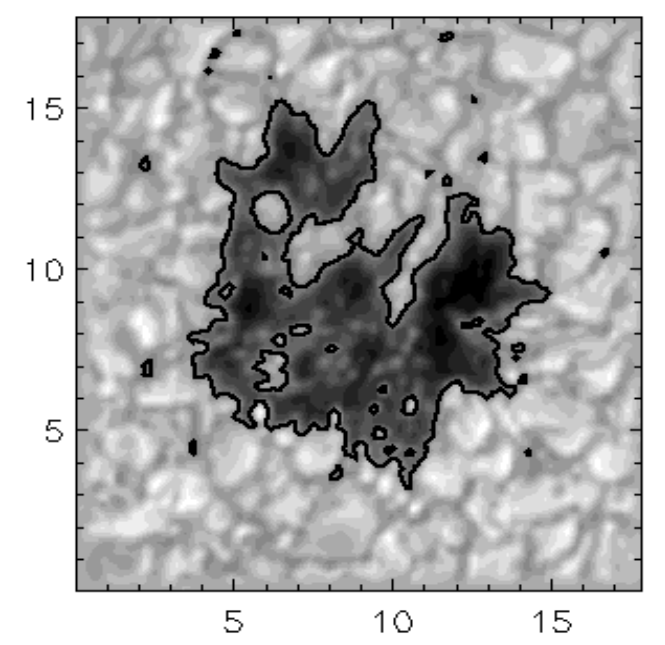

Fig. 1. Image of the pore. The umbra of the pore is outlined by a continuous line corresponding to the iso-intensity level 0.75 of the mean photospheric intensity. Scales are in seconds of arc.

\section{Horizontal velocities outside and inside the pore}

In order to avoid the low spatial frequencies in the intensity fluctuations, as well as the lack of precision ascribed to the LCT technique when used in areas with high intensity gradients (Strous 1995), we applied the LCT method to segmented binarized images (see Roudier et al. 1999). These images are produced by determining the concavity along the intensity field. Those regions where the concavity is downwards are set to 1 and define granules and umbral dots. The rest of the pixels are set to 0 . This segmentation method was proposed by Strous (1994).

The maps of average velocities were computed using a Gaussian window of $F W H M=0,31$ for local correlation and a window of $5 \mathrm{~min}$ for time averaging. Thus, from the whole time series lasting 73 min we produced a datacube consisting of 14 velocity maps $\left(V_{x}, V_{y}, t\right)$, separated in time by a constant interval of $5 \mathrm{~min}$. The small windows in space and time enable more detail in the evolution of plasma motions to be followed quantitatively.

Nevertheless, we have to keep in mind that, in this case, the noise in the velocity determination is higher than for large integration windows, and that the observed small-amplitude velocities might not represent the plasma flow accurately (see Rieutord et al. 2001).

Figure 2 shows an example of three consecutive horizontal velocity maps over the full field of view. The arrows indicate the flow directions and their lengths represent the speed of the flow. The border of the pore is defined as the iso-intensity line corresponding to 0.75 of the mean photospheric intensity. This plot reveals that the inward motion into the pore is abruptly reduced in magnitude by a factor of 2 to 3 within a distance of about $230 \mathrm{~km}$ (the resolution limit of the tracking), as measured from the border inwards into the pore. This effect is not due to the lack of features near the border and inside the pore many features are well visible in the binarized data. This result confirms that of Keil et al. (1999), who found a trend for flows to weaken as magnetic field flux increases. However, unlike the authors mentioned above, we did not observe dark features moving away from pores. This could be because of the evolutionary phase of our pore, which is larger and older than the pore observed by them.

The velocities of the proper motions, averaged over the 5 min windows, range from 0 to $2.2 \mathrm{~km} \mathrm{~s}^{-1}$ with a distribution peaking around $0.3 \mathrm{~km} \mathrm{~s}^{-1}$, as shown in Fig. 3 .

With this spatial resolution in the velocity maps, we observed a diverging activity (small exploders) in the light bridge visible $42.5 \mathrm{~min}$ and $62.5 \mathrm{~min}$ after the beginning of the time series (see Fig. 4). We also observed an outflow from the light bridge into the umbra. The latter result has already been reported by Hirzberger et al. (2002). These authors tracked individual granules in the light bridge by eye to determine their proper motions.

\subsection{Flow patterns outside the pore}

A constant intensity threshold ( 0.75 of the mean photospheric intensity) was selected visually to outline the border of the pore throughout the time sequence. This allows the shape modifications of the pore's umbra to be taken into account. In the previous work by Sobotka et al. (1999), where the same pore was studied, a ringlike structure of positive divergence ("rosettas") was found around the pore in a flow map averaged over the whole time sequence. We confirm this phenomenon with our measurements, made with higher temporal and spatial resolutions. Indeed, for smaller averaging intervals of $5 \mathrm{~min}$ we also observe a ring of positive divergence in the individual maps (see Fig. 2), which is related to a continuous activity of exploding events in the granulation around the pore throughout the time sequence.

The mean distance that we have measured between the centres of the rosettas around the pore is $2^{\prime \prime} \cdot 3 \pm 0$. . This value has to be compared with 2 ". $6 \pm 11^{\prime \prime} 0$, the mean distance that we obtain between the rosettas in the quiet Sun. The difference between 

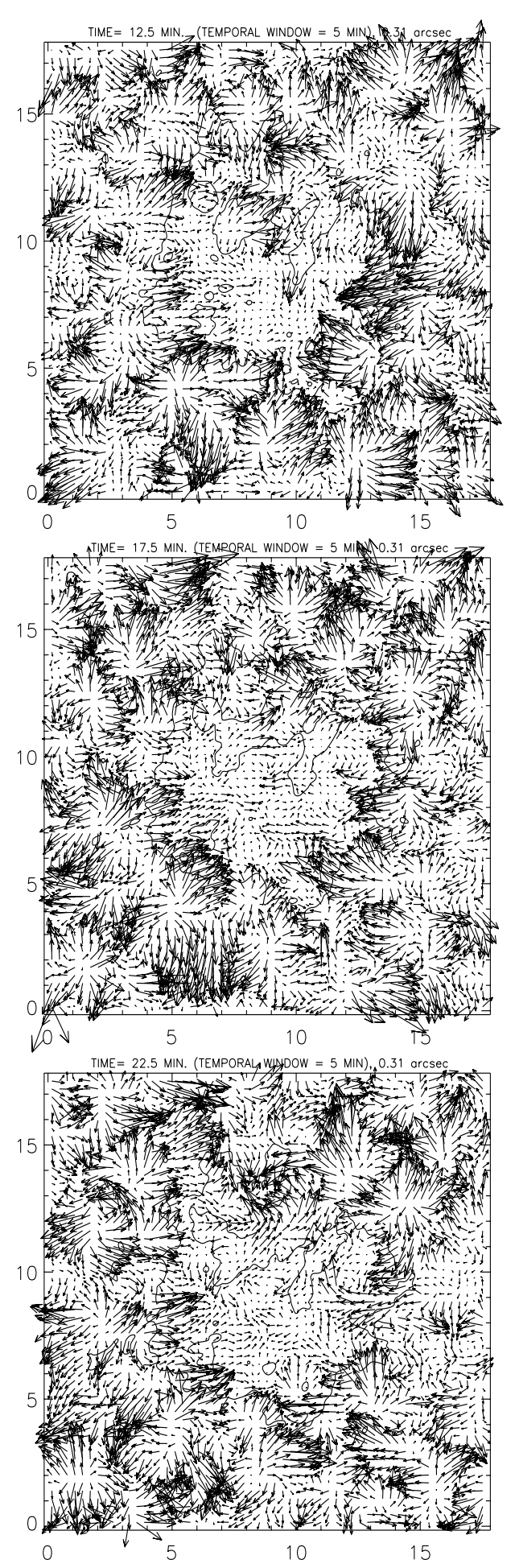

Fig. 2. Three examples of horizontal velocity fields measured with 0.31 resolution and each one averaged over $5 \mathrm{~min}$. The time step between two consecutive maps is $5 \mathrm{~min}$. The umbra of the pore is outlined by the continuous line. Spatial scales are in seconds of arc.

these mean values is only $12 \%$ but the distribution of distances is narrower in the case of the rosettas around the pore than in the quiet Sun, which indicates a more regular mesh of exploding events around the pore.

The magnitudes of the proper-motion velocities outside the pore range from 0 to $2.2 \mathrm{~km} \mathrm{~s}^{-1}$ and the distribution is peaked
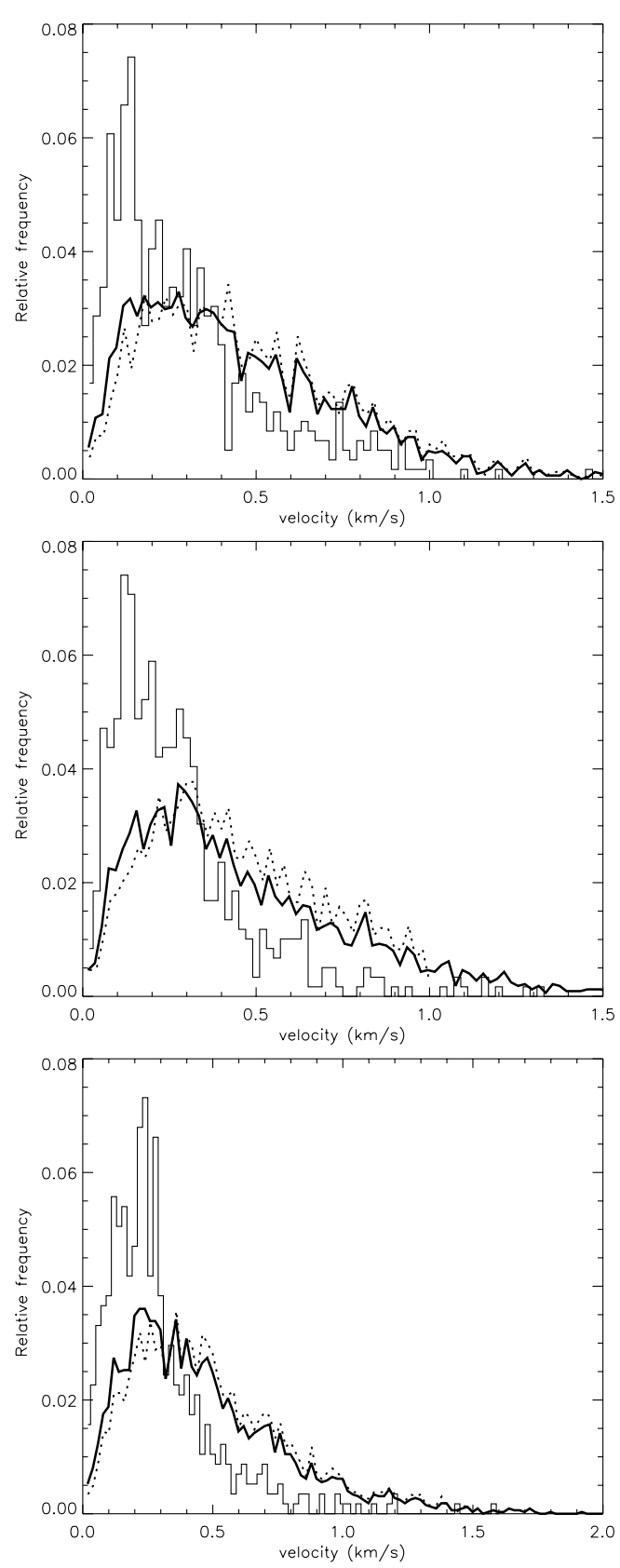

Fig. 3. Histograms of horizontal velocity magnitudes (thick solid line) corresponding to the three maps shown in Fig. 1. The distributions peak around $0.3 \mathrm{~km} \mathrm{~s}^{-1}$ and the velocities reach values as high as $2.2 \mathrm{~km} \mathrm{~s}^{-1}$. The histograms of velocity magnitudes outside (dotted line) and inside (thin solid line) the pore are overplotted.

around $0.35 \mathrm{~km} \mathrm{~s}^{-1}$ with a significant contribution of higher velocities (see Fig. 3).

\subsection{Flow patterns inside the pore}

Figure 5 shows the flow maps inside the pore, averaged over the 5 min temporal windows, for the same examples presented in Fig. 2. Inside the pore, the magnitudes of the velocities are smaller by a factor of 2 to 3 relative to the velocities of the plasma outside the pore (see Fig. 3). 


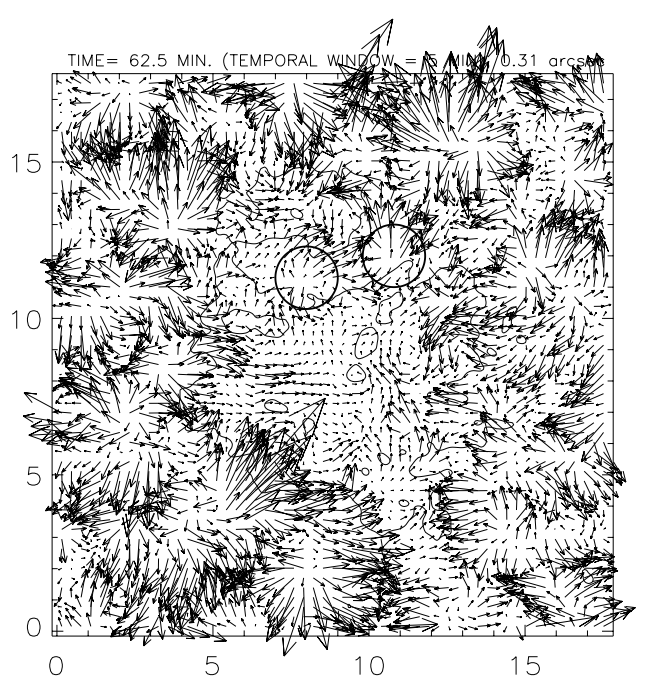

Fig. 4. Two exploding events in the light bridge (marked by circles).

The histogram of velocity magnitudes inside the pore is narrower than that outside the pore. The magnitudes of the velocities range from 0 to $1.3 \mathrm{~km} \mathrm{~s}^{-1}$ with a mean of $0.29 \mathrm{~km} \mathrm{~s}^{-1}$ and their distribution peaks around $0.15-0.20 \mathrm{~km} \mathrm{~s}^{-1}$. This mean velocity is smaller than $0.32 \mathrm{~km} \mathrm{~s}^{-1}$, the mean velocity of umbral dots, determined by Sobotka et al. (1999) using the technique of tracking individual features. This difference can be explained as follows. The window for local correlation in the LCT method includes umbral dots as tracers of the motion but also a fraction of surrounding dark background with zero velocity. The consequence is a kind of averaging of the velocity field by the LCT method and lower velocity magnitudes of the umbral structures. The time-averaged vectors of horizontal velocities of 135 umbral dots with lifetimes longer than $5 \mathrm{~min}$, determined by the feature tracking algorithm, is shown in Fig. 6. We can see that many umbral dots move in the directions indicated by the LCT flow maps in Fig. 5. A detailed description of the feature tracking technique can be found in Sobotka et al. (1997) and Sobotka et al. (1999).

The highest velocities inside the pore are located close to the pore's border and our measurements clearly show a penetrating flow from outside (see Fig. 5). This flow can be seen in all 14 independently calculated velocity maps and corresponds to the penetration of small granules and granular fragments from the photosphere into the pore, pushed by granular motions originating in the divergence centres (rosettas) around the pore (Sobotka et al. 1999). We can conclude from our measurements and in accordance with Sobotka et al. (1999) that the motions at the periphery of the pore are substantially and continuously influenced by the external plasma flows deposited by the explosive events.

\section{Variation of the flows}

The 14 velocity maps separated by the constant time interval of 5 min make it possible to study the changes in the horizontal motions in time. A visual inspection of the maps has shown that the divergence centres (rosettas) around the pore have no
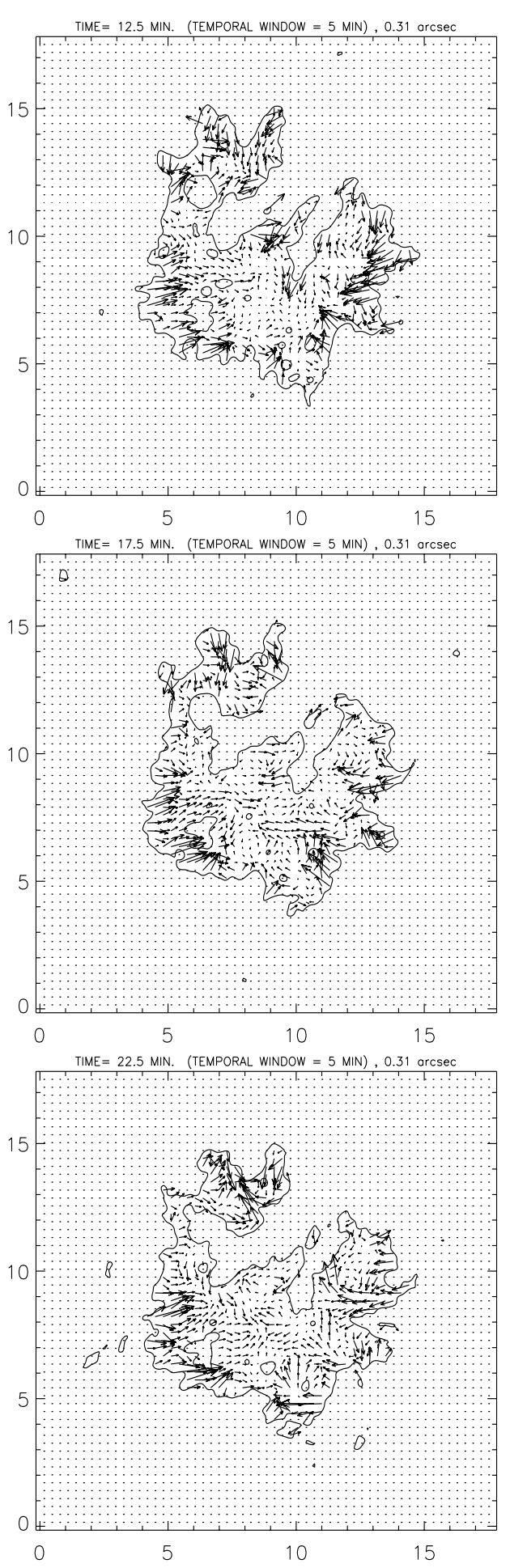

Fig. 5. Horizontal velocity fields inside the pore, corresponding to the three flow maps shown in Fig. 2.

organized motion, rather, they tend to appear recurrently around certain locations.

The temporal properties of the flow fields can be characterized by variations of areas where the velocity magnitudes are greater than a certain threshold. These areas were determined in each map for the threshold speeds $0.3,0.4$, and $0.5 \mathrm{~km} \mathrm{~s}^{-1}$. In Fig. 7 we show, as an example, the locations of such areas (marked in black) for the fourth map $(t=17.5 \mathrm{~min})$. We can see 


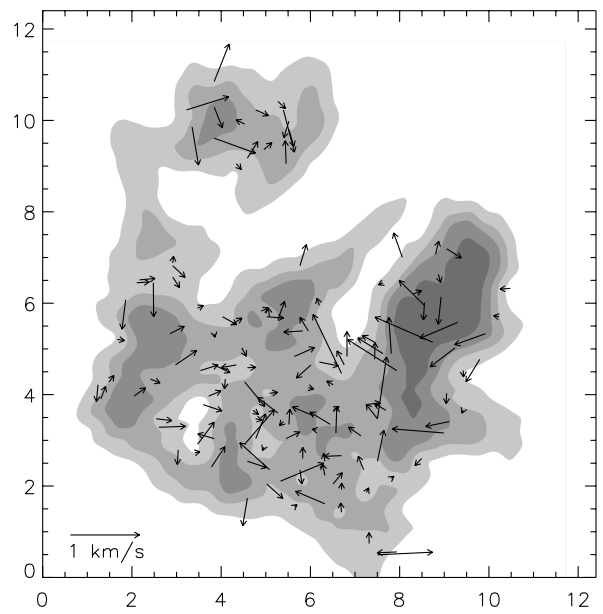

Fig. 6. Vectors of time-averaged horizontal velocities for 135 umbral dots with lifetimes longer than $5 \mathrm{~min}$. The origins of vectors are located at time-averaged positions of umbral dots. The underlying greyscale image of the pore is the average of 220 frames of the series with contours corresponding to intensities $0.4,0.5,0.6$, and 0.75 of the mean photospheric intensity. Scales are in seconds of arc.

that they can mostly be found in the photospheric granulation and at the boundary of the pore.

The temporal variations in the ratio $R$, defined as the sum of the areas with speeds greater than a given threshold divided by the total area of the field, are plotted in Fig. 8 for each threshold value separately. The temporal evolution of the rms intensity of the granulation, a measure of the seeing-dependent image quality, is also shown in the plot. The presented rms intensities were averaged over the same time periods as used to compute the velocity maps. It can be seen from Fig. 8 that, independently of the velocity threshold, we detect fluctuations in $R$ with a characteristic time of 20-30 min that are uncorrelated with the changes of the image quality.

A similar characteristic time (23 min) was found by Sobotka et al. (1999) for quasi-periodic intensity variations of umbral dots inside the pore. We can speculate that repeating (quasi-periodic) changes in the velocity magnitudes of exploding events located around the pore might be somehow connected to the intensity variations of UD's.

To test for the possible influence of the horizontal velocities on the intensity field, we selected in the datacube $\left(V_{x}, V_{y}, t\right)$ such points inside the pore where the velocity magnitude is greater than $0.6 \mathrm{~km} \mathrm{~s}^{-1}$. These points are mostly located close to the border of the pore. For each of these points we estimated the phase delay, $\delta t$, between the temporal variations of velocity, $V(t)$, and intensity, $I(t)$. This phase delay was measured directly from plots of such variations at a given point as the difference between the instants of the maximum amplitude of $V(t)$ and the nearest maximum amplitude of $I(t)$. Only positive $\delta t$ were measured so in that case the velocity variations precede the intensity ones.

We performed a total of 387 measurements and estimated the mean error to be $\pm 5 \mathrm{~min}$. When a measurement was too ambiguous, the $\delta t$ value was discarded. In Fig. 9 is plotted the
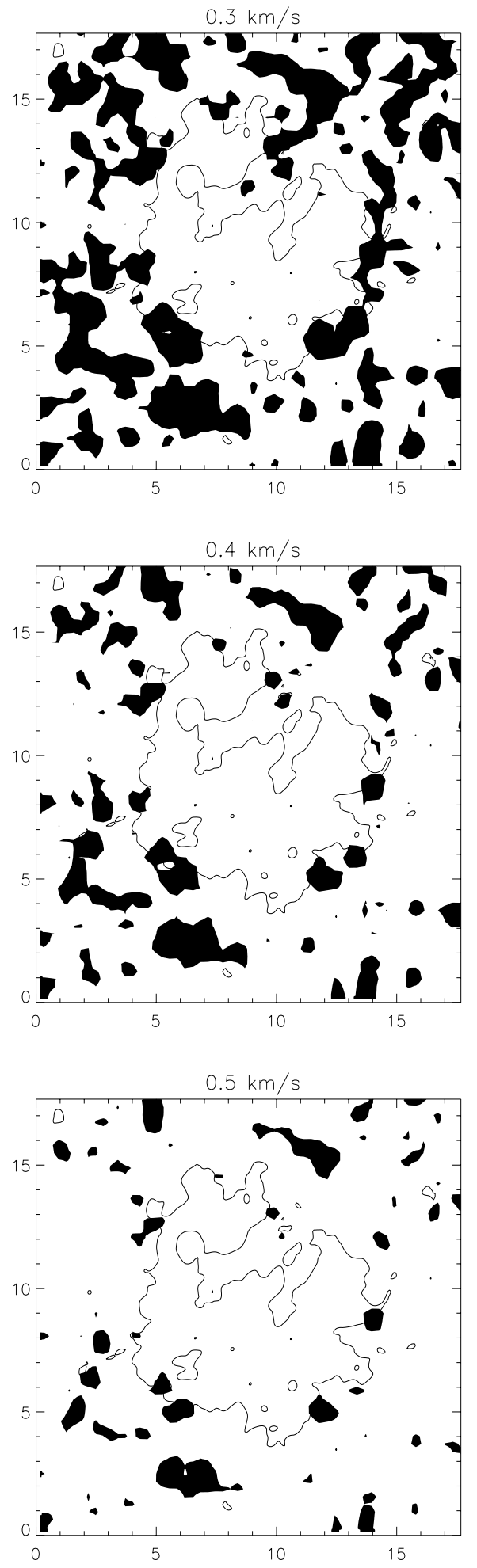

Fig. 7. Locations of areas where the magnitudes of horizontal velocities are greater than $0.3,0.4$, and $0.5 \mathrm{~km} \mathrm{~s}^{-1}$ (from top to bottom).

histogram of all the phase delays, showing a mode around 10$15 \mathrm{~min}$.

This phase delay appears as a quasi-systematic effect in the brightest structures in the pore close to its border. This could be related to the bright features penetrating into the pore, where the velocities increase before intensities. The velocity could 


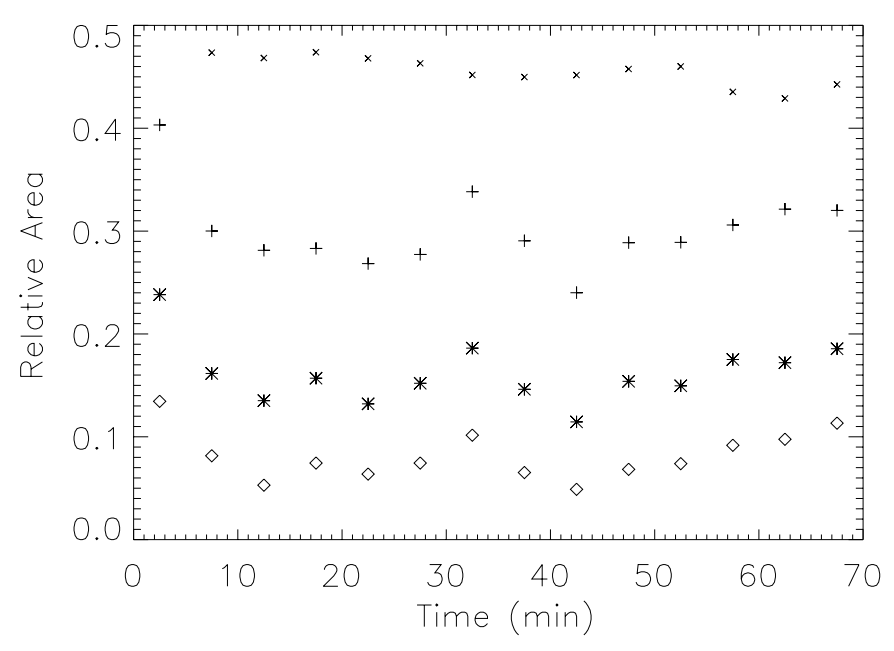

Fig. 8. Temporal variations in the relative area $R$, where the magnitudes of the horizontal velocities are greater than 0.3 (crosses), 0.4 (stars), and 0.5 (diamonds) $\mathrm{km} \mathrm{s}^{-1} ; \times$ symbols represent the $\mathrm{rms}$ intensities of quiet granulation (in arbitrary units), averaged over time periods used for the velocity computations.

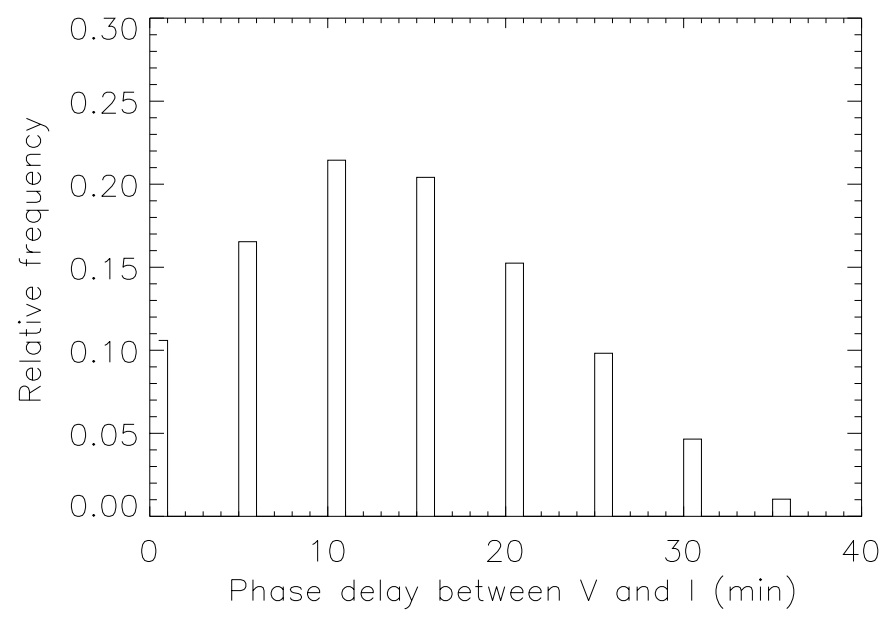

Fig. 9. Histogram of the phase delays between the velocity and intensity variations inside the pore.

be associated with some compression effect in the surrounding material that would induce some back-heating of the moving feature. This would imply only individual physical points in the comparison of intensity and velocity and could justify some time delay. The variation in brightness may also be induced by a kind of collision or buffeting produced by a neighbouring element placed at a certain distance. This mechanism would also justify a time delay (due to a wave propagation time?) in the intensity with respect to the velocity. But this event would imply two different physical points in the pore or in its surroundings.

The 14 velocity maps $\left(V_{x}, V_{y}, t\right)$ have been used to generate cork evolution (November \& Simon 1988). The corks are taken as the positions of these passive scalars at the end of the time sequence. Corks start to conglomerate around the pore at a

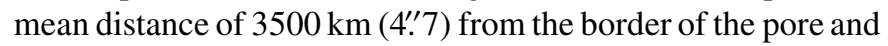
in some of the radial interstices between the explosive events (mesoscale). The corks also outline the boundary of the pore (velocities directed toward the pore) and the boundaries of divergence rosettas.

Due to low-velocity magnitudes, the corks within the pore are still visible everywhere, with no significant concentration. The study of horizontal velocities inside the pore by means of corks would require a longer time sequence.

\section{Discussion and conclusions}

The LCT technique applied to the series of 220 binarized images allowed us to follow simultaneously the velocity fields in the interior of the pore, at the pore's boundary, and in the surrounding photosphere. In this way, in spite of the fact that the LCT velocity measurements have a mostly qualitative character, it is possible to observe flows that are coherent in independent maps and to show the influence of explosive events in the granulation around the pore on the motions of UDs in the peripheral parts of the pore's umbra.

At the boundary of the pore, in the locations without intrusions of bright features into the pore, a very steep gradient of the horizontal velocity is measured. Continuous explosive events around the pore are observed, with a mean distance of 2 '. 3 between them and with a more regular mesh than in the quiet granulation. This is in agreement with the previous result obtained by Sobotka et al. (1999). The dense distribution of explosive events around the pore could be due to the deflection of the ascending plasma by the magnetic field of the pore. A link between the variations (characteristic time of 20-30 min) in the large velocity amplitudes around the pore and the fluctuations in brightness of umbral dots is suggested.

A phase delay between velocity and intensity maxima at the periphery of the pore, probably related to the penetration of bright features inwards across the pore's border, was measured. Its distribution peaks around 10-15 min. This phase delay could be due to different phenomena such as compression effect in the surrounding material that would induce some heating into the moving feature, or the intensity variation could be induced by a kind of collision or buffeting produced by a neighbouring element located at a certain distance corresponding to the observed phase delay. To clarify the effects of external flows on the brightness (temperature) of umbral dots and to study the relations between the velocity and intensity variations at the periphery of the pore, longer time sequences are required, together with Doppler and magnetic field measurements.

Acknowledgements. We wish to thank to D. Kennerdahl and G. Hosinsky for their support during the observations. We thank the Scientific Editorial Service of the Instituto de Astrofísica de Canarias for the revision of the English text. The Swedish Vacuum Solar Telescope is operated on the island of La Palma by the Swedish Academy of Sciences at the Spanish Observatorio del Roque de los Muchachos of the Instituto de Astrofísica de Canarias. This work was partially financed by a) the Centre National de la Recherche Scientifique (CNRS, UMR 5572) and the Programme National Soleil Terre (P.N.S.T.); b) the Spanish Ministerio de Ciencia y Tecnología, project PNAYA2001-1649; c) the Academy of Sciences of the Czech Republic (Key Project K 2043105) and its Grant Agency (grant A 3003903). 


\section{References}

Hirzberger, J., Bonet, J. A., Sobotka, M., Vázquez, M., \& Hanslmeier, A. 2002, A\&A, 383, 275

Keil, S. L., Balasubramaniam, K. S., Smaldone, L. A., \& Reger, B. 1999, ApJ, 510, 422

Molowny-Horas, R., \& Yi, Z. 1994, in Oslo IDL Software for Image Processing and Data Analysis, Internal Report No. 31, Institute of Theoretical Astrophysics, University of Oslo

November, L. J., \& Simon, G. W. 1988, ApJ, 333, 427

Rieutord, M., Roudier, T., Malherbe, J. M., \& Rincon, F. 2001, A\&A, 357,1063

Roudier, Th., Rieutord, M., Malherbe, J. M., Vigneau, J. 1999, A\&A, 349,301
Scharmer, G., Brown, D. S., Pettersson, L., \& Rehn, J. 1985, Appl. Opt., 24, 2558

Simon, G. W., Brandt, P. N., November, L. J., Shine, R. A., \& Strous, L. H. 1995, in Proc. of the 4th SOHO Workshop: Helioseismology, ed. J. T. Hoeksema, V. Domingo, B. Fleck, \& B. Battrick, ESA SP-376, 223

Sobotka, M., Brandt, P. N., \& Simon, G. W. 1997, A\&A, 328, 682

Sobotka, M., Vázquez, M., Bonet, J. A., Hanslmeier, A., \& Hirzberger, J. 1999, ApJ, 511, 436

Strous, L. H. 1994, Ph.D. Thesis (Utrecht)

Strous, L. H. 1995, in Proc. of the 4th SOHO Workshop: Helioseismology, ed. J. T. Hoeksema, V. Domingo, B. Fleck, \& B. Battrick, ESA SP-376, 219 